\title{
A New Approach to Improve the Parameter Estimation Accuracy in Robotic Manipulators Using a Multi-Objective Output Error Identification Technique*
}

\author{
C. West, A. Montazeri, S.D. Monk, D. Duda, C.J. Taylor
}

\begin{abstract}
The research behind this article primarily concerns the development of mobile robots for nuclear decommissioning. The robotic platform under study has dual, seven-function, hydraulically actuated manipulators, for which the authors are developing a vision based, assisted teleoperation interface for common decommissioning tasks such as pipe cutting. However, to improve safety, task execution speed and operator trainingtime, high performance control of the nonlinear manipulator dynamics is required. Hence, the present article focuses on an associated dynamic model, and addresses the challenging generic task of parameter estimation for a highly non-convex and nonlinear system. A novel approach for estimation of the fundamental parameters of the manipulator, based on the idea of multi-objectivization, is proposed. Here, a single objective output error identification problem is converted into a multiobjective optimization problem. This is solved using a multiobjective genetic algorithm with non-dominated sorting. Numerical and experimental results using the nuclear decommissioning robot, show that the performance of the proposed approach, in terms of both the output error index and the accuracy of the estimated parameters, is superior to the previously studied single-objective identification problem.
\end{abstract}

\section{INTRODUCTION}

For several decades robots have been used to replace human workers in manufacturing environments [1], performing repetitive or dangerous jobs, as well as in hostile and dangerous environments such as subsea [2], mining [3] and nuclear [4], where the environment is unsuitable for humans to operate. Traditionally robots have mainly operated in structured environments, such as automotive production lines. Increasingly, however, they are now being used in unstructured and dynamically changing environments. This necessitates the development of smarter, faster and more flexible robotic systems, with the capability to show humanlike characteristics such as sensing, memory, dexterity and trainability. Progress in this area includes the development of semi-autonomous and tele-operated systems, where the human interacts with the robot in a way that assures safe and reliable operation for both manipulator and operator.

To support research and development in this area, a mobile robotic platform, with dual seven degree of freedom (DOF) hydraulically actuated manipulators, has been developed at Lancaster University (see e.g. [5] and Fig. 1). This platform is primary being developed to accomplish specific tasks related to the decommissioning, maintenance and repair of nuclear power facilities. It consists of a BROKK-40 mobile base, to which two HYDROLEK manipulators have been attached. To replace the traditional direct joystick system

\footnotetext{
* The authors are grateful for the support of the National Nuclear Laboratory and Nuclear Decommissioning Authority in the UK.

All the authors are with the Engineering Department, Lancaster University, United Kingdom (corresponding author: a.montazeri@lancaster.ac.uk)
}

used previously, a vision based, assisted teleoperation human machine interface (HMI) is under development by the present authors [6]. This uses a camera to obtain RGB video and 3D depth data for the scene in front of the manipulators. The system presents a straightforward graphical user interface (GUI) to the operator, who with just four mouse clicks can select target positions for each manipulator to e.g. perform a pipe grasp and cut action.

However, designing high performance control algorithms that compensate for the nonlinear dynamics of this manipulator system requires a reliable model that accurately captures the dynamic behaviour of the system [7-8]. This further necessitates development of techniques to estimate the parameters of the model using experimental data under different operating conditions. The importance and challenge of this task in the context of robotics is considered by many authors including, for example, research into trajectory optimization [9] and equation error parameter estimation [10-11]. The major problems in estimation using equation error methods result from the uncertainties due to the modelling errors and measurement noise in the observation matrix. To address this, one approach is to use output error identification. However, as discussed in section 3, this leads to a challenging non-linear and non-convex optimization problem, solved here using a multi-objectivization concept.

Section 2 of the article briefly reviews the robotic platform and assisted teleoperation HMI. Section 3 provides the background to the generic optimisation problem, which is investigated in section 4 by sensitivity analysis. This leads to a new formulation of the estimation problem in section 5 , using the multi-objectivization concept and associated Genetic Algorithm (GA) methods. In section 6, the parameters of the proposed multi-objective GA are tuned for the best performance and the estimation results are compared with a simple GA, as applied to the decommissioning robot. This is followed in section 7 by the conclusions.

\section{HUMAN-MACHINE INTERFACE}

The previously developed robotic platform, illustrated in Fig. 1 and described in more detail by references $[5,7,8]$, has some similarities to the Hitachi Astaco-Sora system [12]. The Hitachi consists of two hydraulic manipulators on a tracked vehicle and is teleoperated using a complex user interface. This complexity arises from the many cameras and sensors, as well as from the teleoperated control system. By contrast, the assisted teleoperation system developed by the present authors, is designed to keep the user engaged and in control at all times, whilst being as straightforward as possible. A multiple camera approach is not always practical in a nuclear decommissioning environment anyway, where 


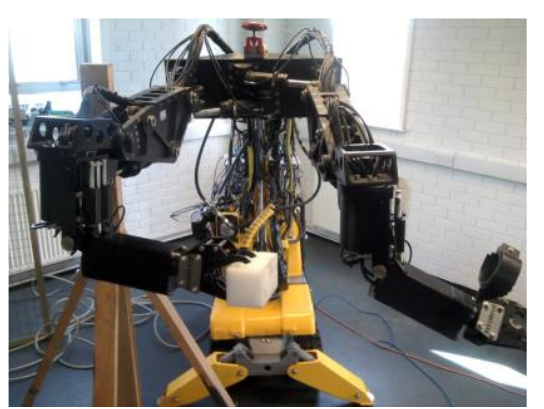

(a)

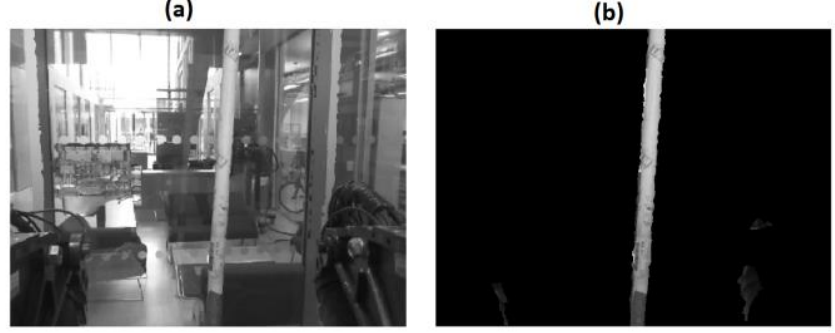

Fig. 1 Top: BROKK-based nuclear decommissioning robot. Lower photos: imagine processing: (a) original and (b) simplified image of the target pipe.

electronics are easily damaged by radiation. In general, it is preferable to reduce the number of sensors and other electronics, limiting the amount of scene information that can be collected and creating restrictions that need to be considered when developing assisted teleoperation.

Due to such limited sensor data availability, a system that can grasp generic objects would be unreliable. As a result, the developed vision system is based on the concept of multiple subsystems for common tasks under one user interface. This includes, for example, one subsystem for pipe cutting and another for pick and place operation. This approach reduces the complexity of the problem, potentially leading to improved performance and reliability. Furthermore, cognitive workload is generally reduced by tailoring the information shown to the user to one particular decommissioning task.

Preliminary work has focused on pipe cutting as an illustration of the generic approach (Fig. 1), since this is a common repetitive task in decommissioning. The user selects the object to be cut from an on-screen image with a mouse click, whilst the computer control system determines the required position and orientation of the manipulators in 3D space, and calculates the necessary joint angles for the manipulator to grasp the object. The approach has some similarities to recent work by Kent et al. [13]. However, testing of the new interface, even in a laboratory environment, has identified the need for improved control systems to provide more accurate movement of the hydraulic manipulators, leading to the research in the present article.

\section{OPTIMISATION BACKGROUND}

One approach to address the nonlinear and non-convex nature of the performance index used for parameter estimation in an output error format, is to use multi-start gradient-based search techniques. The major problem with such techniques is their dependency on the initial conditions. For manipulator parameter estimation, the problem can be alleviated using a closed-loop technique [10]. Another major problem for the present robot, however, is that unlike in references [10-11], calculating the gradient of the performance index with respect to the unknown parameters is not possible analytically: lack of an analytical model prohibits analytical derivation of the gradients, whilst using a performance index such as the infinity norm of the output error leads to a non-differentiable performance index. Other approaches include evolutionary-based optimization, such as differential evolution (DE), particle swarm optimization (PSO) and GAs.

Reference [14] presents an overview of evolutionary algorithms for system identification and filtering. In the context of multi-objective evolutionary optimization, [15] suggests that none of these approaches have been applied in a system identification framework. In fact, quite a few attempts are reported to use multi-objective optimization for the trade-off between the order of the model and the quality of the identification. However, the motivation for the present article is quite different. Here, we use the concept of multiobjectivization [16] to convert a single-objective output error identification problem into a multi-objective one. We have formulated the optimization problem to be solved using the well-known NSGA-II algorithm. Many multi-objective evolutionary algorithms use the idea of non-dominant sorting similar to NSGA-II. GAs are also used by [17-18] for tuning the model parameters of a 3-DOF serial manipulator powered by electric motors and gearboxes. A multi-objective GA is also used in [19] to estimate and tune the parameters of a PID controller for a 2-DOF robot arm. However, other options include replacing the GA with PSO or DE.

\section{PROBLEM DESCRIPTION}

The present article builds on the work in [21, 22] and [20] that used a simple GA in relation to estimating the unknown parameters of the model in robotic application, and further develops the results presented in [8] in using multi-objective GA, with the aim to improve both the parameter estimation accuracy and the output error performance index of the problem. Joint 2 ('shoulder' elevation) is used as an example. In contrast to these earlier articles, to find the minimum set of parameters suitable for estimation, a comprehensive first order identifiability analysis is first discussed. Note that the dynamic model of the manipulator is not described below because of space constraints: the equations and implementation form are in references [7-8].

\subsection{Sensitivity Analysis}

To find a suitable set of parameters for optimisation, an identifiability analysis of the dynamic parameters of the manipulator model was conducted. The sensitivity of the cost function $J_{N}(\boldsymbol{\theta})$ defined later in (2) is calculated with respect to the nine mechanical system model parameters in vector $\boldsymbol{\theta}$. Since no analytical model of the robot dynamics is available, derivatives of the cost function with respect to these parameters are calculated numerically using 
perturbation analysis. The local sensitivity of the cost function $J_{N}(\boldsymbol{\theta})$ is investigated with respect to random changes of individual parameters of the system. Each of the nine parameters are randomly perturbed around the nominal value, with a variance of 0.1 and 1.0 , and the ratio of change in the cost function output over change in parameter value, $\Delta J_{N}(\boldsymbol{\theta}) / \Delta \boldsymbol{\theta}$, determined. For each variance, the average for each parameter over 100 perturbations is shown in Table 1.

The second stage of the sensitivity analysis was to evaluate the sensitivity of the model output $\hat{y}(k ; \boldsymbol{\theta})$ for different values of the parameters, observed by plotting them numerically. This is achieved by changing the value of one parameter at a time to cover the full range of possible values and plotting the model output. Figures 2 and 3 show the simulation output for varying joint spring stiffness and damping coefficient respectively.

Table 1 - Average sensitivity of the performance index for random perturbations. The parameters are defined in [7-8].

\begin{tabular}{|c|c|c|}
\hline Parameter & Variance 0.01 & Variance 0.1 \\
\hline Gain & 23.303 & 1803.014 \\
\hline Damping & 19251.758 & 1803.868 \\
\hline Spring Stiffness & 23010.938 & 1817.792 \\
\hline Mass 1 & 24420.943 & 1841.055 \\
\hline Mass 2 & 25777.600 & 1921.174 \\
\hline Mass 3 & 26938.055 & 1929.087 \\
\hline Mass 4 & 27339.732 & 2085.842 \\
\hline Mass 5 & 249642.931 & 2166.694 \\
\hline Mass 6 & 250412.766 & 2212.637 \\
\hline
\end{tabular}

Fig. 2 Simulation output (Joint 2 angle) for varying the spring stiffness from $5 \mathrm{mN} / \mathrm{rad}$ to $100 \mathrm{mN} / \mathrm{rad}$.

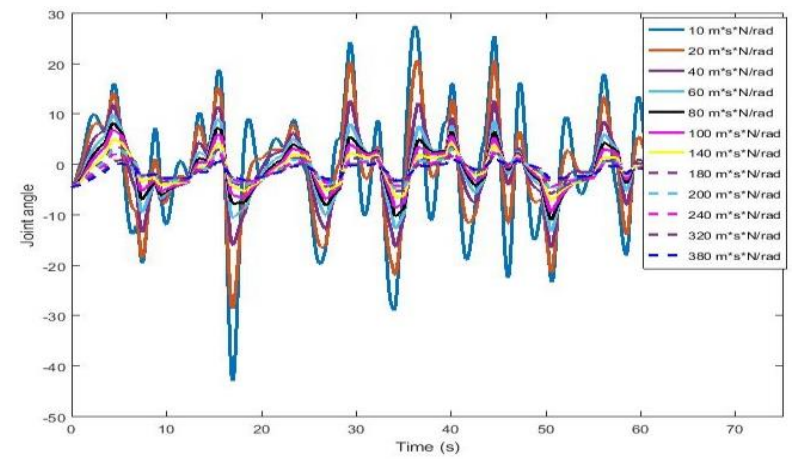

Fig. 3 Simulation output (Joint 2 angle) for varying the damping coefficient from $10 \mathrm{msN} / \mathrm{rad}$ to $400 \mathrm{msN} / \mathrm{rad}$.
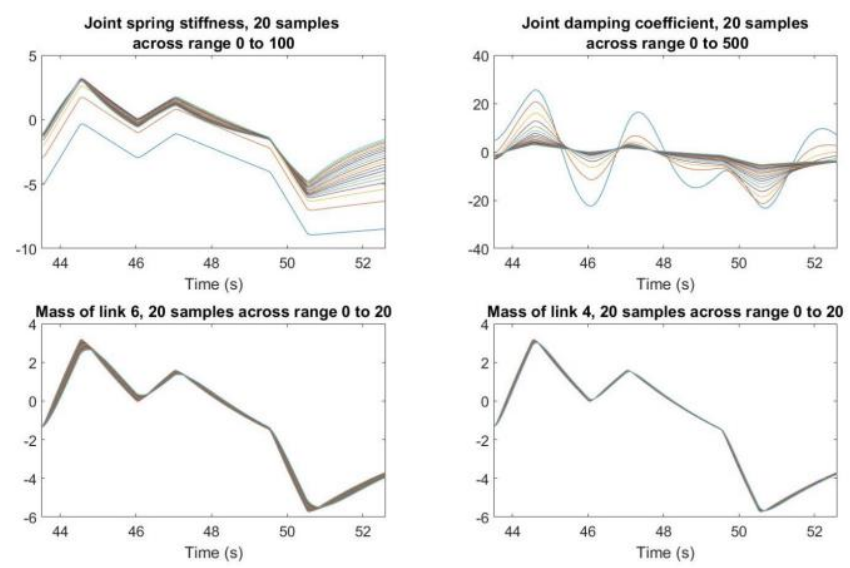

Fig. 4 Comparison of the sensitivity of the simulation output to four parameters (i.e. spring stiffness, damping coefficient and the mass of links 4 and 6) over the same time segment.

Sensitivity is much lower for the link masses, with the mass of link 6 being the most sensitive and sensitivity decreasing towards link 1. This result is in agreement with the data in Table 1. An important point to observe is that the sensitivity of the output, as shown in Figures 2 and 3, is a function of time. For some time segments, the output is more sensitive to change of parameters than for other time segments. As is evident from Fig. 2, the output is less sensitive to parameter changes around the starting time and becomes increasingly sensitive as time goes on. Alternatively, by looking at the sensitivity of different parameters in an identical time frame, as shown in Fig. 4, it is possible to obtain a better idea of how the output sensitivity differs for various parameters.

We exploit this property in minimization of the performance index (2), by splitting the model output into a number of segments and treating each segment as a separate objective for the multi-objective GA. One advantage of using multiple segments is that it introduces cross validation in parallel to parameter estimation when the GA is running. For each segment, the estimated parameters are validated against the fitness value for other segments. A good parameter set in the sense of the multi-objective performance index should have a low fitness value across all segments. Since, in the ideal case, there is only one parameter set that ensures the minimum fitness value across all segments, i.e. the global optimum solution, it is expected that the algorithm achieves an improved convergence to the optimum parameter values in this way. Finally, note that a similar analysis is performed for the parameters of the hydraulic actuator subsystem.

\subsection{Performance Surfaces}

Another measure that provides an improved understanding for selection of a suitable optimization algorithm is the nature of the performance surface. To obtain a visual understanding of the type of non-linearity and non-convexity of the problem, the performance surface is plotted as a function of two exemplar parameters from Table 1 . Examples of these surfaces are shown in Figures 5 and 6. 


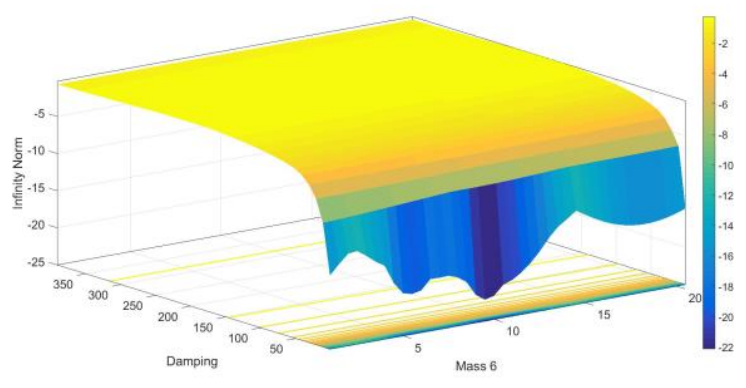

Fig. 5 Performance surface showing the infinity norm of the cost function as a function of the mass of link 6 and the damping coefficient.

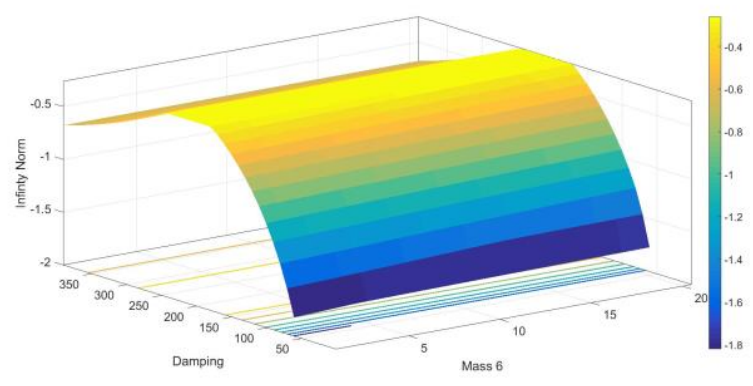

Fig. 6 Performance surface showing the infinity norm of the cost function as a function of mass of link 6 and damping coefficient, with a smaller range of output error than Fig. 5, hence showing the non-flat surface.

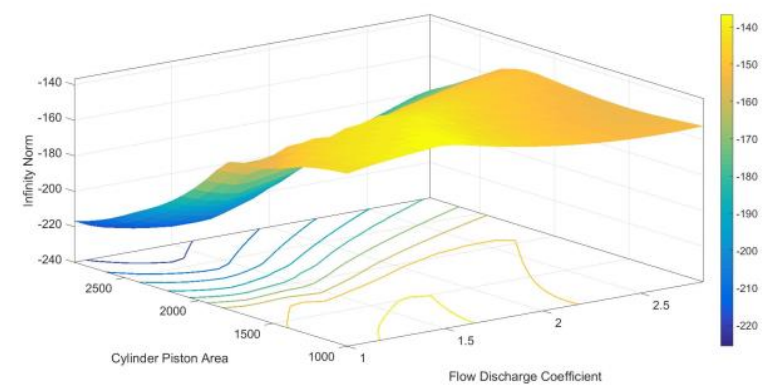

Fig. 7 Performance surface showing the infinity norm of the output error as a function of the flow discharge coefficient and cylinder piston area for the hydraulic actuator.

Fig. 5 shows the infinity norm of the performance index (2) as a function of the damping coefficient and Mass of link 6 . A 'zoomed-in' version of this plot around the optimum values is shown in Fig. 6. These figures show that the performance index has an almost flat surface around a range of values for the desired parameters, which makes the estimation problem a difficult task. This result demonstrates the non-convex nature of the estimation problem. As will be shown later, by using the concept of multi-objectivization of the performance index, it is possible to find performance indices that are more sensitive to the unknown parameters and hence result in a better overall estimation performance. The performance surface to estimate the parameters of the hydraulic actuator with respect to the piston area and flow discharge coefficient is shown in Fig. 7. The surface for the hydraulic parameters is more irregular than the mechanical parameters, revealing more information on the nonlinearity of the optimization problem. Finally, Fig. 5 also justifies the use of GA methods due to their parallel processing capabilities and hence capability for climbing steep surfaces.

\section{ESTIMATION PROBLEM FORMULATION}

The mechanical model parameters to be estimated are listed in Table 1 and represented by a vector $\boldsymbol{\theta}$. The excitation input used for estimation is the voltage sent to the hydraulic actuator system for a single joint $i$, i.e. $u_{i}(k)$, and the output is the joint angle measured by the potentiometer at joint $i$, i.e. $y_{i}(k)$. To ensure that realistic parameter values are found by the algorithm, the search space $\boldsymbol{\Omega}$ is defined in a way to include all prior knowledge about the physical system. As an example, the search space for the mechanical subsystem $\Omega_{M} \subset \mathbb{R}^{9}$ is defined as:

$\Omega_{M}=\left\{\boldsymbol{\theta} \mid \boldsymbol{\theta}=\left(\theta_{1}, \theta_{2}, \ldots, \theta_{9}\right), \quad \theta_{\min _{i}}^{M} \leq \theta_{i} \leq \theta_{\max _{i}}^{M} i=\right.$ $1,2, \ldots 9\}$

To formulate the single objective output error system identification problem into a multi-objective optimisation problem, the output signal is split into several segments, and a separate objective function is defined for each segment. The cost function $J_{N_{S}}^{S}(\boldsymbol{\theta})$ for output segment $s$ is defined as the $p$-norm of the absolute error signal for $p=1,2, \infty$ :

$J_{N}(\boldsymbol{\theta})=\left(\frac{1}{N} \sum_{k=1}^{N}\left|\mathcal{E}_{i}^{S}(k ; \boldsymbol{\theta})\right|^{p}\right)^{\frac{1}{p}}$

The identification error in (2) is calculated for each segment $s$ of joint $i$ defined as:

$\mathcal{E}_{i}^{S}(k ; \boldsymbol{\theta})=y_{i}^{s}(k)-\hat{y}_{i}(k ; \boldsymbol{\theta})$

where $\hat{y}_{i}(k ; \boldsymbol{\theta})$ represents the model output at joint $i$ for a specific parameter vector $\boldsymbol{\theta}$ and $y_{i}^{S}(k)$ is the measured output at segment $s$. The multi-objective cost function defined as a result of the multi-objectivization is:

$J_{\text {mul }}(\boldsymbol{\theta})=\left[J_{N_{1}}^{1}(\boldsymbol{\theta}), J_{N_{2}}^{2}(\boldsymbol{\theta}), \cdots, J_{N_{n}}^{n}(\boldsymbol{\theta})\right]$

where $n$ is the number of objectives, in this case the number of segments the measured output is split into. To judge the quality of each vector $\boldsymbol{\theta}$, the fitness value for each segment is calculated separately. The optimal solution for each segment $s$ is determined by selecting the parameter vector with the minimum fitness value, hence using the cost function in equation (2), the optimal estimated parameter vector $\widehat{\boldsymbol{\theta}}_{\mathrm{N}}$ is:

$\widehat{\boldsymbol{\theta}}_{\mathrm{N}}=\arg \min _{\boldsymbol{\theta} \in \Omega_{M}} \frac{1}{N} \sum_{k=1}^{N}\left|\mathcal{E}_{i}^{S}(k ; \boldsymbol{\theta})\right|^{p}$

After the GA has completed a set number of iterations, a population containing the best solution is found. From this the optimal $\boldsymbol{\theta}$ is found for each segment, yielding $n$ sets of parameters. The average of each parameter is subsequently taken to yield the final compromised solution. 


\section{The Proposed Multi-OBJective GenetiC Algorithm}

The algorithm proposed to estimate the parameter vector $\boldsymbol{\theta}$ is based on the NSGA-II algorithm using non-dominant sorting and Pareto optimal solutions. The fitness function (2) for $p=\infty$ shows the best results, similar to the simple GA approach [24]. For effective use of the multi-objective GA, its parameters should be tuned for this particular problem. For this purpose a set of known parameters for the simulation model is assumed and the model output is used for tuning the multi-objective GA. To study the effect of different GA parameters, such as crossover, mutation, and population size, the data are split into eight segments and the relative parameter estimation error, as well as the sum of square errors, for each case are calculated and compared.

Table 2 shows the results of the investigation into the crossover value, and the final set of estimated parameters are compared. The sum of relative errors for different estimated parameters is adopted as an indication of performance in Table 2. Similar results are generated for the population size, mutation rate, and number of segments but are not reported here due to lack of space. Table 2 suggests that a unity crossover rate yields the best performance in terms of estimation accuracy. However, since the goal is to improve the parameter estimation accuracy, along with a good output match, a compromise is necessary.

The final values of the tuned parameters for both the simple GA and the new multi-objective GA are listed in Table 3 . Finally, Table 4 compares the estimation results for the simple GA and multi-objective GA using two and eight segments. Comparison of the relative estimation error for the proposed methods shows that the multi-objective with eight segments yields the best results.

\section{Conclusions}

The research behind this article concerns the development of an assisted teleoperation HMI for nuclear decommissioning tasks such as robotic pipe cutting. The article has focused on the parameter estimation problem, using a multi-objective GA, for a nonlinear mechanistic model of a dual manipulator robotic platform. The dynamic model of the device was developed in earlier articles [7-8]. The results in the present article have increased the accuracy of the model, so that the advanced control systems needed to improve the semiautonomous operation of the device can be developed.

In particular, by inspection of the performance surface and sensitivity of the measured output with respect to different parameters, it is possible to find a suitable multiple objective performance index, for which the parameter estimation accuracy will be improved. The key concept is to split the single objective output error performance index into a multiobjective performance index using a multi-objectivization concept; and subsequently to determine the estimated parameters from the pareto-optimal solution of the algorithm. Numerical results show that the new approach performs better than a simple GA studied previously for the same problem [20]. This involves a better accuracy in parameter estimation as well as lower values for the output error estimation.

Although not the focus of the article, the vision based, assisted teleoperation system alluded to in section 2, provides the main motivation for this research. The new system has recently been tested using the decommissioning robot in a laboratory environment [6]. It is shown to work successfully, outperforming the currently used joystickbased teleoperation approach, when tested with both experienced and inexperienced operators. Preliminary experiments suggest that standard tasks such as grasping a pipe are completed faster, and the user requires less training, in comparison to the traditional teleoperation approach. However, control of the nonlinear hydraulic manipulator dynamics needs improving and, for example, one limitation of the present prototype is the lack of a sophisticated collision avoidance system, something that is presently being developed using the dynamic model from this article.

\section{REFERENCES}

[1] Zhang, G. Q., Li, X., Boca, R., Newkirk, J., Zhang, B., Fuhlbrigge, T A., Feng, H. K. \& Hunt, N. J. 2014. Use of Industrial Robots in Additive Manufacturing - A Survey and Feasibility Study. 41st International Symposium Robotics.

[2] Chantler, M. \& Reid, C. 1991. Sensor fusion of range and intensity data for subsea robotics. 5th Int. Conference on Advanced Robotics.

[3] Konyukh, V. 2002. Robotics for Mining. Mineral Resources Engineering, 11 (73).

[4] Bogue, R. 2011. Robots in the nuclear industry: a review of technologies and applications. Industrial Robot: An International Journal, 38, 113-118.

[5] Taylor, C. J. \& Robertson, D. 2013. State-dependent control of a hydraulically actuated nuclear decommissioning robot. Control Engineering Practice, 21, 1716-1725.

[6] West, C., Montazeri, A., Monk, S. D., Duda, D. \& Taylor, C. J. 2017. Semi-autonomous vision based pipe cutting system for a mobile dual manipulator nuclear decommissioning robot (submitted)

[7] Montazeri, A., Ekot J.U. 2016. Development of dynamic model of a 7DOF hydraulically actuated tele-operated robot for decommissioning applications. American Control Conference, Boston, MA., USA.

[8] Montazeri, A., West, C., Monk, S. D. \& Taylor, C. J. 2016. Dynamic modelling and parameter estimation of a hydraulic robot manipulator using a multi-objective genetic algorithm. International Journal of Control, 90, 661-683.

[9] Swevers, J., Ganseman, C., De Schutter, J. \& Van Brussel, H. 1996 Experimental Robot Identification Using Optimised Periodic Trajectories. Mechanical Systems and Signal Processing, 10, 561-577.

[10] Gautier, M., Janot, A. \& Vandanjon, P.-O. 2013. A new closed-loop output error method for parameter identification of robot dynamics. IEEE Transactions on Control Systems Technology, 21, 428-444.

[11] Janot, A., Vandanjon, P.-O. \& Gautier, M. 2014. A generic instrumental variable approach for industrial robot identification. IEEE Transactions on Control Systems Technology, 22, 132-145.

[12] Kinoshita, H., et al. 2014. Development of new technology for Fukushima Daiichi nuclear power station reconstruction. Hitachi Review, 63, 183 .

[13] Kent, D., Saldanha, C. \& Chernova, S. 2017. A Comparison of Remote Robot Teleoperation Interfaces for General Object Manipulation. ACM/IEEE International Conference on Human-Robot Interaction, Vienna.

[14] Gotmare, A., Bhattacharjee, S. S., Patidar, R. \& George, N. V. 2016. Swarm and evolutionary computing algorithms for system identification and filter design: A comprehensive review. Swarm and Evolutionary Computation.

[15] Marler, R.T., Arora, J.S. 2004. Survey of multi-objective optimization methods for engineering. Structural and Multidisciplinary Optimization, 26, 369-395. 
[16] Lochtefeld, D. F. \& Ciarallo, F. W. 2015. Multi-objectivization Via Decomposition: An analysis of helper-objectives and complete decomposition. European J. of Operational Research, 243, 395-404.

[17] Grecu, V., Grecu, L., Demian, G., Demian, M., Mastorakis, N. \& Martin, O. A. 2009. Genetic Algorithm for Optimization in Conceptual Robot Manipulator. WSEAS International Conference Mathematics and Computers in Science and Engineering, Rodos.

[18] Jafari, A., Safavi, M. \& Fadaei, A. 2007. A Genetic Algorithm to Optimum Dynamic Performance of Industrial Robots in the Conceptual Design Phase. 10th IEEE International Conference on Rehabilitation Robotics, Noordwijk.
[19] Ayala, H.V.H \& Coelho, L. 2012. Tuning of PID controller based on a multiobjective genetic algorithm applied to a robotic manipulator. Expert Systems with Applications, 39, 8968-8974.

[20] West, C., Montazeri, A., Monk, S. D. \& Taylor, C. J. 2016. A genetic algorithm approach for parameter optimization of a 7DOF robotic manipulator. 8th IFAC Conference on Manufacturing Modelling, Management and Control, Troyes.

[21] Montazeri, A., Poshtan, J., 2009. GA-based optimization of a MIMO ANC system considering coupling of secondary sources in a telephone kiosk. Applied Acoustics, 70 (7), pp. 954-953.

[22] Montazeri, A., Poshtan, J., 2009. Optimizing a multi-channel ANC system for broadband noise cancellation in a telephone kiosk using genetic algorithms. Shock and Vibration, 16(3), pp. 241-260.

Table 2 - Effect of crossover on the performance of the multi-objective GA.

\begin{tabular}{|c|c|c|c|c|c|c|c|c|c|c|}
\hline \multirow{2}{*}{ 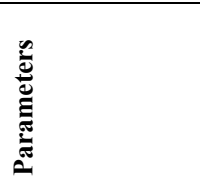 } & \multirow[b]{2}{*}{ گే } & \multirow{2}{*}{ 䓂 } & \multirow[b]{2}{*}{$\stackrel{\mathscr{E}}{\stackrel{E}{E}}$} & \multicolumn{6}{|c|}{ Mass of link (kg) } & \\
\hline & & & & 1 & 2 & 3 & 4 & 5 & 6 & \\
\hline True. & 0.2 & 231.8 & 53.4 & 4.6 & 5.1 & 22.5 & 1.6 & 4.0 & 4.7 & \\
\hline Crossover 1.0 & 0.36 & 314.27 & 39.06 & 2.97 & 4.99 & 16.08 & 5.78 & 7.12 & 6.99 & \\
\hline Crossover 0.8 & 0.19 & 214.27 & 45.11 & 3.61 & 9.05 & 21.90 & 8.08 & 15.23 & 6.18 & \\
\hline Crossover 0.6 & 0.27 & 293.03 & 59.93 & 3.31 & 10.38 & 20.04 & 9.14 & 16.19 & 13.29 & \\
\hline Crossover 0.4 & 0.19 & 214.27 & 45.11 & 3.61 & 9.05 & 21.90 & 8.08 & 15.23 & 6.18 & \\
\hline \multicolumn{10}{|c|}{ Relative Error } & Sum \\
\hline Crossover 1.0 & 0.8 & 0.36 & 0.27 & 0.35 & 0.02 & 0.28 & 2.6 & 0.78 & 0.48 & 5.94 \\
\hline Crossover 0.8 & 0.05 & 0.08 & 0.15 & 0.22 & 0.77 & 0.02 & 4.05 & 2.81 & 0.31 & 8.46 \\
\hline Crossover 0.6 & 0.35 & 0.26 & 0.12 & 0.28 & 1.03 & 0.10 & 4.7 & 3.05 & 1.83 & 11.72 \\
\hline Crossover 0.4 & 0.05 & 0.08 & 0.15 & 0.21 & 0.77 & 0.03 & 4.05 & 2.81 & 0.31 & 8.46 \\
\hline
\end{tabular}

Table 3 - GA settings used to generate the parameter estimation results in Table 4.

\begin{tabular}{|c|c|c|}
\hline Setting & Simple GA Value & Multi-objective GA Value \\
\hline Coding scheme & Multivariable binary coding & Multivariable binary coding \\
\hline Crossover rate $(\mathrm{Pc})$ & 0.8 & 0.4 \\
\hline Mutation rate $(\mathrm{Pm})$ & 0.05 & 0.2 \\
\hline Parent selection & proportional & proportional \\
\hline Crossover type & pointwise & pointwise \\
\hline Population size & 70 & 20 \\
\hline Fitness function & infinity norm & infinity norm \\
\hline
\end{tabular}

Table 4 - Evaluation of the effect of segmentation on the accuracy of parameter estimation.

\begin{tabular}{|c|c|c|c|c|c|c|c|c|c|}
\hline \multirow[b]{2}{*}{ 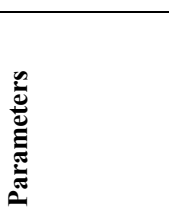 } & \multirow[b]{2}{*}{ שี } & \multirow[b]{2}{*}{ 禺 } & \multirow[b]{2}{*}{$\stackrel{\mathscr{E}}{\mathscr{E}}$} & \multicolumn{6}{|c|}{ Mass of link (kg) } \\
\hline & & & & 1 & 2 & 3 & 4 & 5 & 6 \\
\hline True. & 0.2 & 231.8 & 53.4 & 4.6 & 5.1 & 22.5 & 1.6 & 4.1 & 4.7 \\
\hline Simple GA & 0.4 & 343.2 & 89.4 & 4.6 & 10.60 & 0.80 & 10.0 & 19.5 & 5.9 \\
\hline 2 segments & 0.38 & 430.60 & 79.27 & 3.29 & 9.05 & 22.33 & 9.55 & 15.02 & 13.26 \\
\hline 8 segments & 0.1 & 112.4 & 37.5 & 2.3 & 2.3 & 13.3 & 5.1 & 2.2 & 4.5 \\
\hline \multicolumn{10}{|c|}{ Relative Error } \\
\hline Simple GA & 1 & 0.48 & 0.67 & 0 & 1.08 & 0.96 & 5.25 & 3.76 & 0.26 \\
\hline 2 Segments & 0.9 & 0.86 & 0.48 & 0.28 & 0.77 & 0.01 & 4.97 & 2.66 & 1.82 \\
\hline 8 Segments & 0.5 & 0.52 & 0.29 & 0.5 & 0.55 & 0.41 & 2.19 & 0.46 & 0.04 \\
\hline
\end{tabular}

